\title{
Diffraction efficiency enhancement of transient holographic gratings in nematic liquid crystals doped with Disperse Red 1
}

\author{
Hongjing $\mathrm{LI}^{1, \text { a }}$, Changshun WANG ${ }^{1, b_{*}}$ \\ ${ }^{1}$ State Key Lab of Advanced Optical Communication Systems and Networks, \\ Department of Physics and Astronomy, Shanghai Jiao Tong University, Shanghai, 200240, China \\ aemail: Ihjnet2012@sjtu.edu.cn, bemail: cswang@sjtu.edu.cn
}

Keywords: Azo dye; Liquid crystals; Holographic grating

\begin{abstract}
The diffraction properties of transient holographic gratings recorded by two $532 \mathrm{~nm}$ laser beams were investigated in nematic liquid crystals doped with Disperse Red 1. A nearly 30-fold enhancement of the first-order diffraction efficiency as a function of heating time was obtained when the sample temperature was close to the clearing point in the nematic phase. A peak shift of diffraction efficiency with the increase of recording light intensity was observed, which was attributed to laser induced photochemical nematic-isotropic phase transition.
\end{abstract}

\section{Introduction}

Azo dye doped nematic liquid crystals have been actively investigated due to their promising applications in display technology, optical storage, optical limiting and nonlinear optical devices [1-4]. Disperse Red 1 (DR1) doped nematic liquid crystals have been regarded as one of the most potential materials for application in transient holographic display because of the fast response time of several milliseconds and no need for external electric field [5-7]. However, the lower diffraction efficiency is not beneficial to the development and its practical application. It is well known that azo dye molecules can undergo trans-cis-trans photoisomerization process after absorbing suitable optical energy, which exerts intermolecular torque to align liquid crystals perpendicular to the polarization of incident light [8-11]. The reorientation of nematic liquid crystals caused by photoisomerization process of azo dye molecules plays an important role in the refractive index modulation of holographic grating. On the other hand, the refractive indices of nematic liquid crystals are closely related with temperature especially near the phase transition temperature.

In this paper, we report the temperature-dependent diffraction efficiency of photo-induced grating in DR1 doped liquid crystals under continuous wave (CW) $532 \mathrm{~nm}$ laser interference. The first-order diffraction signal intensity has an obvious enhancement near the nematic-isotropic phase transition temperature with homogeneous alignment. A peak shift of diffraction efficiency toward lower temperature can be observed with the increase of recording light intensity.

\section{Experiments}

The material used in the current work was a mixture of a nematic liquid crystal (5CB) doped with DR1 molecules. The homogenous mixture was injected into an empty cell by capillary. The sample was sandwiched by two indium-tin oxides (ITO) glass substrates to maintain the cell gap with $50 \mu \mathrm{m}$ thick spacers, and the two ITO glass substrates were precoated with polyvinyl alcohol (PVA) and rubbed in the same direction to obtain homogeneous alignment, which was confirmed using polarizing microscope. The sample has a strong absorption maximum in the visible spectral range near $510 \mathrm{~nm}$ that corresponds to the $n-\pi^{*}$ electronic transition of trans isomer of DR1 molecule [19]. The differential scanning calorimetry (DSC) data showed that the sample's clearing temperature was $35.08^{\circ} \mathrm{C}$. 
(a)

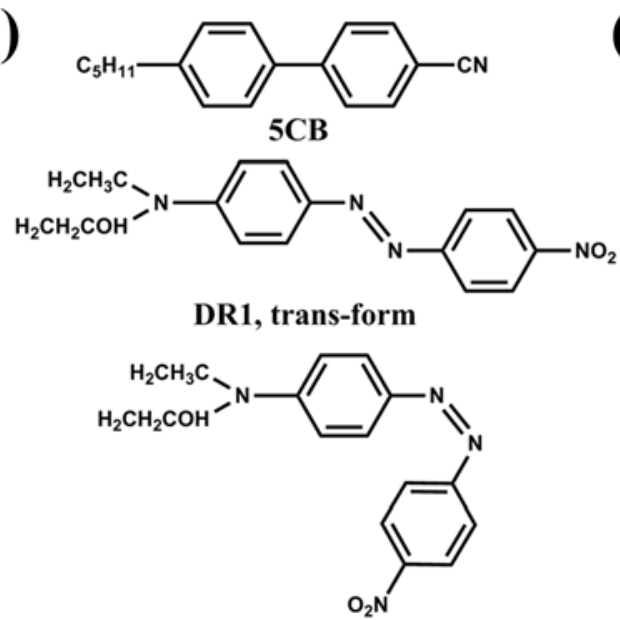

DR1, cis-form

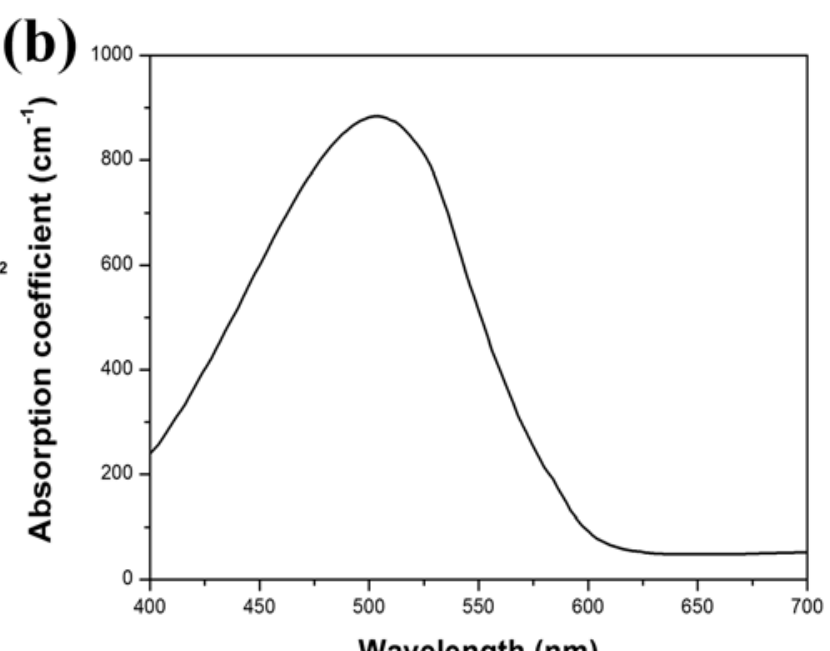

Wavelength (nm)

Figure 1. (a) Chemical structures of 5CB and DR1; (b) absorption spectra of nematic liquid crystals doped with 1 wt. \% DR1.

Figure 2 illustrates the experimental setup for investigating the temperature-dependent diffraction of photoinduced grating. Two s-polarized recording beams with the same diameter of 2 mm were both derived from a CW Nd:YAG laser $(\lambda=532 \mathrm{~nm})$, intersected at an angle of $\theta \sim 3^{\circ}$ in the sample. These two beams had the equal-intensities and were overlapped on the sample. The sample was placed in a temperature-controlled chamber with glass window, to enable the recording beams transmitted. The heating rate of the chamber was precisely controlled at $0.5{ }^{\circ} \mathrm{C} / \mathrm{s}$ by a temperature controller (HCS302, Instec Co.). The intensities of the first-order diffraction with temperature variation were simultaneously detected by a photodetector and recorded by a digitizing storage oscilloscope (Tektronix DPO2014).

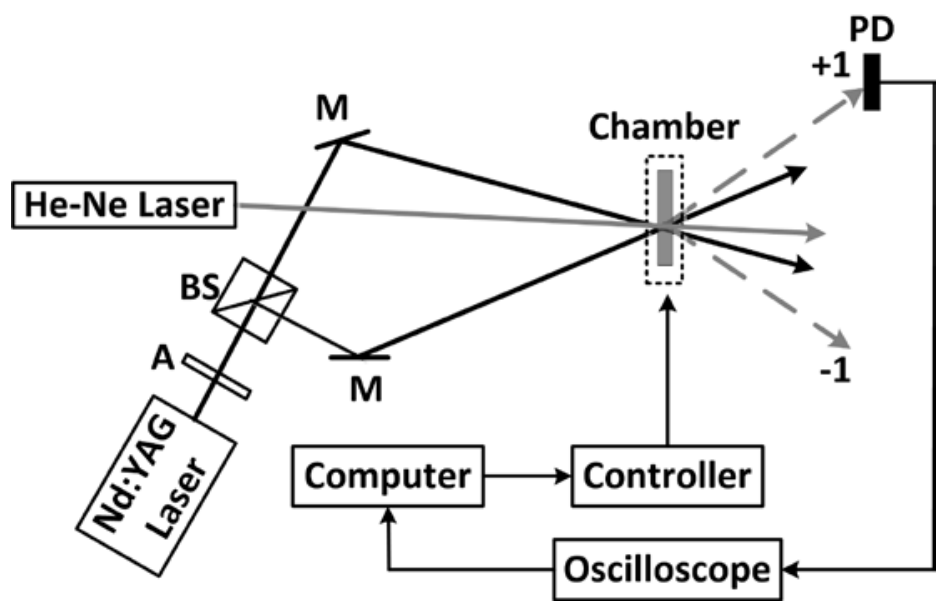

Fig. 2. Schematic diagram of the experimental setup; A: attenuator, BS: beamsplitter, M: mirror, PD: photodetector.

\section{Results and discussion}

Figure 3 shows the first-order diffraction efficiencies as a function of heating time with different recording light intensities. The chamber was heated for $36 \mathrm{~s}$ at $0.5^{\circ} \mathrm{C}$ intervals from $27^{\circ} \mathrm{C}$ to $45^{\circ} \mathrm{C}$. Different recording light intensities, $65 \mathrm{~mW} / \mathrm{cm}^{2}, 128 \mathrm{~mW} / \mathrm{cm}^{2}, 192 \mathrm{~mW} / \mathrm{cm}^{2}, 260 \mathrm{~mW} / \mathrm{cm}^{2}$, were used to investigate the diffraction properties. The dynamic responses of diffraction efficiency to different light intensities showed similar behaviors. For the recording intensity of $260 \mathrm{~mW} / \mathrm{cm}^{2}$, there is a nearly 30 -fold enhancement of the first-order diffraction efficiency was obtained when the chamber temperature was heated up to $34.8^{\circ} \mathrm{C}$. At the same time, second-, third-order diffractions were gradually generated. The typical self-diffraction pattern with the recording intensities of 260 $\mathrm{mW} / \mathrm{cm}^{2}$ at $34.8{ }^{\circ} \mathrm{C}$ is shown in Fig. 4. As the chamber temperature exceeded $34.8{ }^{\circ} \mathrm{C}$, the diffraction efficiency decreased sharply to a stable value, which was smaller than that at room 
temperature.

In order to study the temperature dependence of the first-order diffraction efficiency, we found the minimum transmitted zero-order beam powers are happened when the diffraction efficiency peaks can be observed. It is well known that the transmitted power is the maximum in the isotropic phase where light scattering is minimal. One can deduce the diffraction efficiency peaks are happened near the clearing point in nematic phase. The diffraction efficiency enhancement occurring in the sample near the phase transition is a critical effect, which is accompanied by strong fluctuation of density and order parameter that could lead to large refractive index variation of the sample and enhance the diffraction efficiency of holographic gratings in DR1 doped liquid crystals.

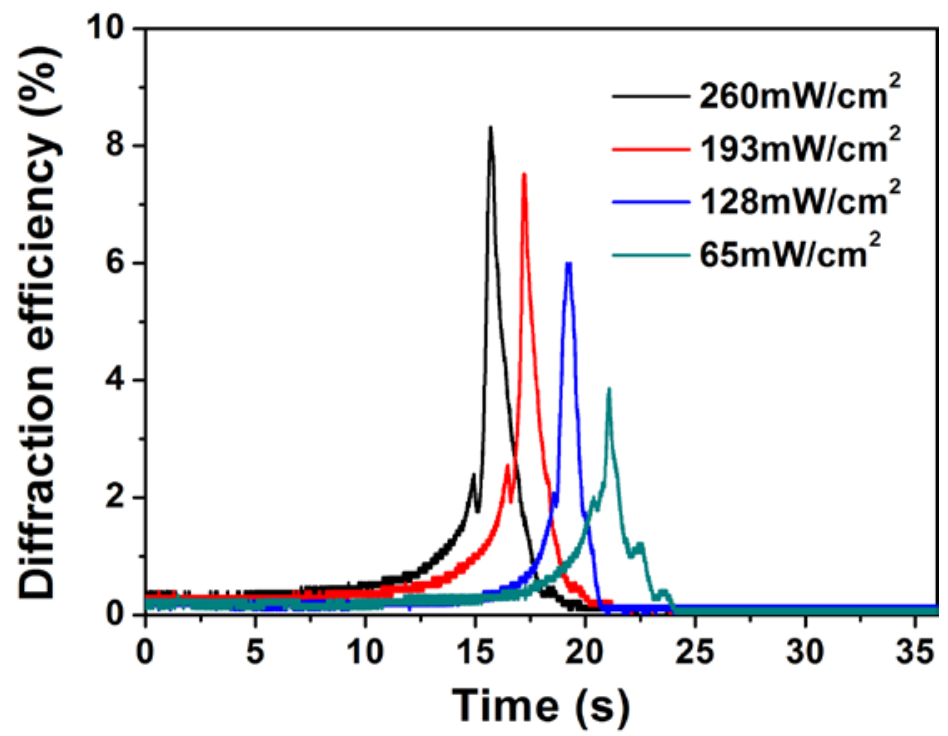

Fig. 3. First-order diffraction efficiencies as function of heating time with different recording light intensities.

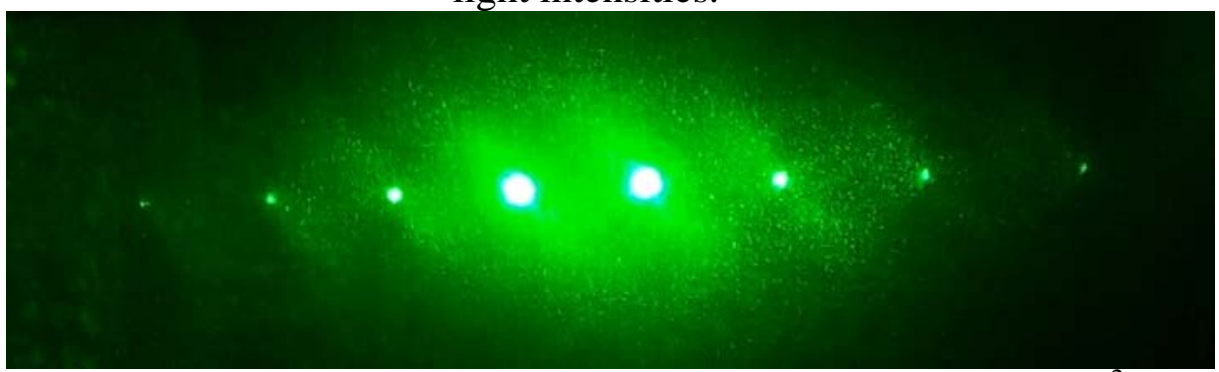

Fig. 4. Diffraction patterns with the recording intensities of $260 \mathrm{~mW} / \mathrm{cm}^{2}$ at $34.8^{\circ} \mathrm{C}$.

There is a peak shift of diffraction intensity toward lower temperature with the increase of recording light intensity in Fig. 3. The temperature variation of the peak diffraction efficiency for 65 $\mathrm{mW} / \mathrm{cm}^{2}$ and $260 \mathrm{~mW} / \mathrm{cm}^{2}$ is about $2.5^{\circ} \mathrm{C}$, indicating that the increasing recording light intensity might result in the sample temperature variation. However, the sample temperature was precisely controlled by the temperature controller. In the experiment, when the recording light intensity is increased, the fraction of cis states of azo dye molecules increases. The reaction of azo dye molecules reduces the order parameter of the nematic phase. The photochemical disordering is increased at elevated temperatures below the clearing temperature of liquid crystal. This effect is similar to the thermal effect.

\section{Conclusion}

We have investigated the diffraction properties of holographic gratings in DR1 doped liquid crystals with homogeneous alignment using two $532 \mathrm{~nm}$ laser beams interference. The first-order diffraction efficiency as a function of heating time in our experiments can reach nearly 30 -fold enhancement when the sample temperature is close to phase transition temperature. The peak shift of diffraction efficiency towards the lower temperature was observed with the increase of recording light intensity, which was attributed to laser induced photochemical disordering. 


\section{Acknowledgement}

This work was supported by the National Natural Science Foundation of China (No. 11574211) and the fund of State Key Laboratory of Advanced Optical Communication Systems and Networks.

\section{References}

[1] L. Lucchetti, J. Tasseva. Optically recorded tunable microlenses based on dye-doped liquid crystal cells [J]. Appl. Phys. Lett. 2012:100 (18): 181111.

[2] C. -R. Lee, T. -S. Mo, K. -T. Cheng, T. -L. Fu, A. Y. G. Fuh. Electrically switchable and thermally erasable biphotonic grating in dye-doped liquid crystal films [J]. Appl. Phys. Lett. 2003:83 (21): 4285-4287.

[3] H. Khoshsima, H. Goodarzi, S. A. Kandijani, R. A. Sabet. The study of dynamic behavior of transient grating in azo dye doped nematic liquid crystal [J]. Mol. Cryst. Liq. Cryst. 2012:560 (1): 62-66.

[4] A. G. Chen, D. J. Brady. Surface-stabilized holography in an azo-dye-doped liquid crystal [J]. Opt. Lett. 1992:17 (17):1231-1233.

[5] Hongjing Li, Jianhao Wang, Changshun Wang, Pengfei Zeng, Yujia Pan, Yifei Yang. Enhanced diffraction properties of photoinduced gratings in nematic liquid crystals doped with Disperse Red 1 [J]. Proceedings of the Japan Academy, Series B, 2016:92 (8):330-335.

[6] Hongjing Li, Jianhao Wang, Changshun Wang, Pengfei Zeng, Peng Cai, Yujia Pan, Yifei Yang. Off-resonant nonlinear optical refraction properties of azo dye doped nematic liquid crystals [J]. Optical Materials Express, 2016:6 (2):459-465.

[7] Y. J. Wang, G. O. Carlisle. Optical properties of disperse-red-1-doped nematic liquid crystal [J]. J. Mat. Sci. Mat. Elect. 2002:13 (3):173-178.

[8] I. Janossy and L. Szabados. Optical reorientation of nematic liquid crystals in the presence of photoisomerization [J]. Phys. Rev. E 1998:58 (4):4598-4604.

[9] I. C. Khoo, H. Li, and Y. Liang. Observation of orientational photorefractive effects in nematic liquid crystals [J]. Opt. Lett. 1994:19 (21):1723-1725.

[10] X. Tong and Y. Zhao. Multiple photochemical processes in liquid crystalline azo dye-doped liquid crystals [J]. Chem. Mater. 2009:21 (17):4047-4054.

[11] L. Lucchetti, M. Gentili, and F. Simoni. Pretransitional enhancement of the optical nonlinearity of thin dye-doped liquid crystals in the nematic phase [J]. Appl. Phys. Lett. 2003:86 (15):151117. 